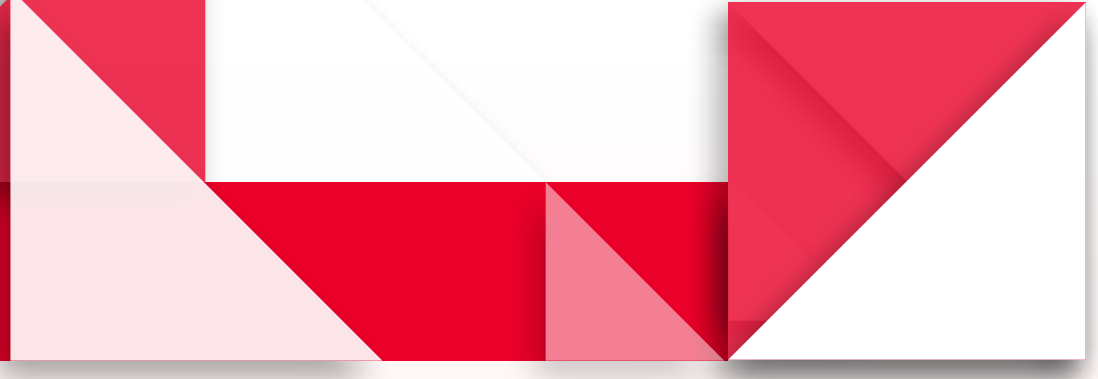

\title{
Investigación e innovación en la enseñanza de las ciencias. Necesidad de una mayor vinculación
}

- Research and Innovation in Science Education: Necessity of a stronger connection

- Investigação e Inovação no Ensino de Ciências: Necessidade de Uma Maior Vinculação

\section{Resumen}

En este artículo se analizan las vinculaciones investigación/innovación en la enseñanza de las ciencias, mostrando resultados que ponen de manifiesto que esta relación no es en la práctica tan estrecha como sería conveniente debido principalmente a dos causas: por un lado, los docentes prestan una escasa atención a la investigación en didáctica de las ciencias e incluso desconocen las revistas especializadas en las que dicha investigación se publica; y, por otro, los investigadores ven la innovación como una mera aplicación de la investigación. Como resultado de estos análisis, se avanza en algunas propuestas para que la investigación contribuya más eficazmente a una mejora generalizada de la enseñanza de las ciencias.

\section{Abstract}

This paper describes the relationship between Research and Innovation in Science Teaching, showing a worrying disconnection of both activities. Two possible causes of this situation are considered: firstly, most teachers do not pay attention to science education research and even ignore the specific journals where this research is published; and secondly, researchers consider innovation as mere application of research. As a result of this analysis, some proposals have been carried out in order to make research more useful to improve science teaching and learning.
Amparo Vilches Daniel Gil Pérez*

* Universitat de València.amparo.vilches@uv.es; daniel.gil@uv.e
Palabras clave:

mejora de la enseñanza de las ciencias, brecha entre investigación e innovación, concepciones docentes, formación del profesorado, alfabetización científica.

Keywords:

Science Education improvement;

Research-Innovation gap; Teacher's Conceptions; Teacher Education; Science Literacy.

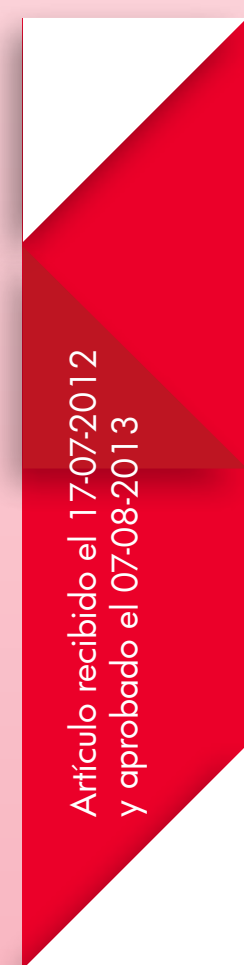




\section{Resumo}

São analisadas as vinculações investigação-inovação no Ensino de Ciências, mostrando resultados que manifestam que esta relação não é tão forte como seria conveniente devido a duas causas: por um lado, os docentes dão pouca atenção à investigação em didática das ciências e, inclusive, desconhecem as revistas especializadas nas quais tais pesquisas são publicadas; e, por outro, os investigadores e investigadoras veem a inovação como uma mera aplicação da investigação. Como resultados destas análises, se apresentam algumas propostas para que a investigação contribua mais eficazmente em uma melhora generalizada do ensino das ciências.
Palavras-chave:

melhora do Ensino das Ciências; Lacuna entre investigação e inovação; Concepções docentes; Formação de professores; Alfabetização científica. 


\section{Introducción}

Preguntarse acerca de las posibles relaciones entre investigación educativa y mejora de la enseñanza de las ciencias podría considerarse una pregunta retórica, ya que, como sabemos, el surgimiento de la didáctica de las ciencias como campo de investigación está ligado fundamentalmente a las necesidades de mejora de la educación científica, derivadas de un doble hecho: la creciente necesidad de científicos y tecnólogos, así como de una alfabetización científica básica para el conjunto de la ciudadanía y el grave fracaso de la enseñanza de las ciencias para lograr estos objetivos (Gil-Pérez, Carrascosa y Martínez, 2000; Anderson, 2007; Rocard et al., 2007).

Se ha comprendido que el aprendizaje y la enseñanza de las ciencias no constituyen actividades sencillas, por lo que se requieren investigaciones sistemáticas en torno a los problemas que plantean y la construcción de un cuerpo coherente de conocimientos que fundamente los cambios necesarios, más allá de innovaciones puntuales y aisladas. En otras palabras, se ha comprendido que la investigación es absolutamente necesaria para una mejora significativa de la educación científica.

Pero esta relación, en la práctica no sigue siendo tan estrecha como parece conveniente $y$, de hecho, podemos encontrar numerosas referencias en la literatura acerca de la escasa influencia que la investigación en didáctica de las ciencias tiene en la enseñanza vigente (Briscoe, 1991; Cronin-Jones, 1991 ; Anderson y Helms, 2001 ; Russell y Martin, 2007).

Los análisis de esta situación se han centrado hasta aquí en estudiar en qué medida los profesores incorporan (o, más bien, no incorporan) los hallazgos de la investigación a su trabajo en el aula. En nuestra opinión, sin embargo, es preciso dirigir también la atención a cómo plantean la relación investigación/ innovación los propios investigadores (Gil-Pérez y Vilches, 2004a). De acuerdo con ello, estudiaremos:

- Cuáles son las percepciones del profesorado acerca del papel de la investigación en la renovación de su docencia.

- Cómo ven los investigadores el papel de la innovación en relación a su trabajo de investigación.

Como resultado de ambos análisis se avanzan algunas propuestas para que la investigación contribuya más eficazmente a una mejora generalizada de la enseñanza de las ciencias.

\section{¿Qué importancia concede el profesorado a la investigación para la mejora de su enseñanza?}

El desconocimiento de una gran parte de docentes de ciencias de las aportaciones de la investigación educativa y, más concretamente, de la investigación en la didáctica de su área, es un hecho que fue reiteradamente señalado en la literatura y que dio lugar a expresiones como research-practice gap, que podemos traducir como "brecha entre la investigación y la práctica en el aula" (Pekarek, Krockover y Shepardson, 1996).

Con objeto de conocer en qué medida esta brecha tiende a disminuir, como sería de desear, o se mantiene en el tiempo, hemos diseñado y utilizado un breve cuestionario para averiguar:

- Las revistas de investigación en didáctica de las ciencias que los profesores manejan o, al menos, conocen. 
- Sus percepciones acerca de las aportaciones de la investigación a la mejora del proceso de enseñanza/aprendizaje (Gil-Pérez y Vilches, 2004a).

Paralelamente hemos realizado entrevistas a docentes de ciencias que están ultimando su formación inicial y también hemos recogido resultados del profesorado en activo de enseñanza secundaria y universidad.

\section{Cuadro 1. Cuestionario para docentes}

Antes de comenzar este curso de didáctica, conviene tomar en consideración qué es lo que ya conocéis acerca de esta materia. A tal fin os rogamos contestéis estas dos cuestiones:

- ¿Qué revistas de didáctica de las ciencias conoces?

- ¿̇Qué aportaciones conoces de la investigación en didáctica de las ciencias para la mejora de la enseñanza?

Los resultados obtenidos mediante el cuestionario (cuadro 1), aplicado a una muestra de 91 docentes en formación y 18 activos, de la Comunidad Valenciana (España), indican que una gran mayoría de profesores encuestados ( $95 \%$ y $72 \%$ respectivamente), incluidos aquellos que habían recibido cursos y talleres impartidos por investigadores en el campo de la didáctica, no conoce revistas de didáctica de las ciencias, limitándose a mencionar algunas de divulgación científica (como Investigación y ciencia, Mundo científico, Muy interesante, Scientific American, etc.) de divulgación educativa (como Cuadernos de Pedagogía) o incluso de investigación en otras áreas científicas (como Inorganic Chemistry). Revistas que, como puede verse, poco tienen que ver con la investigación en didáctica de las ciencias. Además, en algunos casos precisaban que en realidad "les sonaban" pero que nunca las habían leído o utilizado.

En lo que respecta a posibles contribuciones de la investigación para la mejora de la educación científica, la mayoría de los encuestados no menciona ninguna ( $98 \%$ y $83 \%$, respectivamente, de las dos muestras estudiadas). Esta ignorancia resulta, sin duda, preocupante, pues indica que apenas se ha avanzado en la vinculación de la investigación educativa con la enseñanza y la formación del profesorado (Russell y Martin, 2007).

Estos resultados, obtenidos reiteradamente, se ven confirmados en las entrevistas realizadas a docentes en activo para profundizar en el estudio de sus concepciones acerca de la didáctica de las ciencias y del papel de la investigación en la mejora de la enseñanza.

Dichas entrevistas han consistido en tres ítems de preguntas planteadas a 20 profesores activos de física, química, biología y geología, con una experiencia docente de al menos 5 años, de centros educativos de la Comunidad Valenciana:

Ítem 1. ¿Qué revistas de didáctica de las ciencias conoces?

Ítem 2. En todo este tiempo que estás trabajando en la enseñanza, ¿̇has introducido mejoras en tus clases?, ¿̇has cambiado cosas que has visto que no funcionan, has pensado que conviene hacerlo de otra manera? ¿Qué te ha ayudado a la introducción de esas mejoras? ¿̇En qué te has basado? 
Ítem 3. ¿̇ué aportaciones conoces de la investigación en didáctica de las ciencias para la mejora de la enseñanza?

Por lo que se refiere al primer ítem de la entrevista, los resultados son coincidentes con los del cuestionario, ya que casi la totalidad de los entrevistados (18 de los 20 docentes implicados) dice no conocer ninguna revista de investigación o cita revistas de otros ámbitos.

En cuanto a la primera pregunta del segundo ítem de la entrevista ("żhas introducido mejoras en tus clases? Żhas cambiado cosas que has visto que no funcionan, has pensado que conviene hacerlo de otra manera?"), la totalidad de los encuestados reconocen que han ido introduciendo cambios, han intentado mejorar sus clases. Este es, sin duda, un resultado positivo, pues como señalan Simon y Campbell (2012), el deseo de cambiar constituye un requisito imprescindible para la mejora de la enseñanza.

Ahora bien, en lo que respecta a la continuación de las preguntas ("¿̇ué te ha ayudado a la introducción de esas mejoras? ¿En qué te has basado?"), la mayoría de los docentes responde, en esencia, que se han apoyado en su experiencia personal, en lo que han ido probando y han visto si funcionaba bien o no, en su propia reflexión. No hay referencias a la implicación en investigaciones en torno a los problemas encontrados y ni siquiera al estudio de la investigación realizada por otros. Reproducimos, a continuación, un ejemplo de respuesta a estas preguntas:

Pregunta (P). En todo este tiempo que estás trabajando en la enseñanza, żhas introducido mejoras en tus clases? ¿has cambiado cosas que has visto que no funcionan, has pensado que conviene hacerlo de otra manera? ¿¿Qué te ha ayudado a la introducción de esas mejoras? ¿En qué te has basado?
Respuesta (R). Sobre todo en mi experiencia y a veces esa experiencia se ha enriquecido, por decirlo bien, a base de cursos, de comentar con los compañeros, más que de estudiármelo y leérmelo en libros. Sobre todo por la experiencia, la de mis compañeros y la mía.

P. ¿̇Has hecho uso de algo de todo eso para mejorar tus clases? Para mejorar la enseñanza...

R.: Sí, sí, pero no es que lo haya leído. Si en alguna conversación se ha hablado de algo, yo he procurado aplicarlo... que me sale bien o me sale mal, pues vuelvo atrás o sigo adelante. Pero no porque haya cogido un estudio y que diga el tal porcentaje de tal...

\section{P. ¿̇En qué cosas concretas?}

R. En general, en todo. En la forma de dar las clases... En las prácticas mucho, y en la forma de dar la clase, en la forma de enfrentarme... El tipo de explicaciones, la velocidad a la que doy la clase...

P. Y ¿̇cómo has cambiado?

R. Voy más despacio, o más deprisa, depende, sí. En general se va más despacio, y con más cuidado, participar antes también participaban, pero cada vez más. Cada vez participando más los alumnos.

P. Pero, ¿̇ piensas que ha habido una evolución todos estos años?

R. Sí. Haciendo mucho hincapié en sus errores, al participar, intentando sacar sobre todo cuando se equivocan, que además me alegro mucho y digo: "me alegro mucho que me lo hayas dicho mal". Porque me da pie a utilizar sus errores conceptuales 0 , bueno, no conceptuales, errores cualquiera, para avanzar. 
Y he aquí otro ejemplo de respuesta que justifica los cambios haciendo referencia a la realización de cursos:

Pregunta (P). En todo este tiempo que estás trabajando en la enseñanza, żhas introducido mejoras en tus clases?, Żhas cambiado cosas que has visto que no funcionan, has pensado que conviene hacerlo de otra manera?¿̨ué te ha ayudado a la introducción de esas mejoras? ¿̇En qué te has basado?

Respuesta (R). Sí, yo desde que empecé y cuando daba clases en BUP [Bachillerato Unificado Polivalente] daba clases mucho más teóricas, pero desde que empecé con lo de la ESO [Educación Secundaria Obligatoria], sí que he introducido muchos conceptos a nivel didáctico de prácticas, mucha práctica. Me baso más en la práctica y luego los conocimientos teóricos deducidos un poco de la práctica. Prácticas sencillas. Bueno, también un poco guiada por cursos que he hecho en los Cefires [Centro de Formación, Innovación y Recursos Educativos] de didáctica. He hecho cursos de didáctica de las ciencias, también a la hora de plantear los problemas he visto otra forma de ver el planteamiento de problemas y también he hecho cursos que son de esa parte de tutorías, de acción tutorial... Y ves que casi se llega más a ellos pues a través no sé razonando con prácticas y que lo vean. Y luego también recursos de Internet, páginas web para que consulten... Eso también son cursos que he hecho en el Cefire de Sagunto que he hecho tres años de cursos de didáctica a través de medios informáticos, de programas informáticos, de diseñar páginas web...

Por último, los resultados obtenidos en las respuestas al tercer ítem de las entrevistas ("¿Qué aportaciones conoces de la investigación en didáctica de las ciencias para la mejora de la enseñanza?") confirman una vez más el desconocimiento de los docentes entrevistados acerca de las aportaciones de la investigación y su contribución a la mejora de la enseñanza de las ciencias, ya que 18 de los 20 entrevistados no conocen ninguna o citan ejemplos que no tienen que ver con dichas aportaciones. Los resultados de los profesores en activo son, pues, semejantes a los obtenidos con el cuestionario pasado a 98 docentes en formación.

Veamos algunos ejemplos de respuesta obtenidos en la entrevista: "el estudio del movimiento de forma activa por los estudiantes, con gráficas"; "lo más importante es que puedes acceder a Internet"; "la explicación del principio de incertidumbre con una analogía"; "algunos cursos de la Facultad de Física, de contenidos de diferentes temas de física, pero lvego no puedo hacer nada porque no disponemos de material... Seguro que hay cosas muy interesantes pero yo no conozco nada de didáctica".

Es particularmente preocupante el hecho de que algunos de los profesores entrevistados señalen explícitamente que su práctica docente "no debe nada" a la investigación en didáctica de las ciencias. Muestran así no solo el desconocimiento que venimos señalando sino, además, un rechazo cuyas razones deberían ser 
cuidadosamente estudiadas, pero que, según se desprende de algunas argumentaciones expuestas en las entrevistas, pueden asociarse, en parte al menos, al hecho de que dichos profesores ven las propuestas de mejora como una imposición por parte de investigadores y otros expertos, que "trabajan fuera del aula" y desconocen "el mundo real". Reproducimos, a continuación, un ejemplo de respuesta a esta última pregunta:

P. ¿̇Qué aportaciones conoces de la investigación en didáctica de las ciencias para la mejora de la enseñanza?

R. Estoy muy separada de la investigación didáctica... Muy distanciada... No tengo ni idea. Aunque a lo mejor me ha influido, a lo mejor los cursillos que he hecho... Todo ello me ha afectado a la hora de dar mi clase.

P. Pero, más concretamente, ¿̇conoces alguna aportación para la mejora de la enseñanza?

R. Bueno sí, en un cursillo sobre inteligencia emocional... Me afectó mucho, me gustó mucho... Leí un libro y me di cuenta de que cada persona capta las cosas de una forma diferente. Cada uno necesita una forma diferente para aprender. Yo creo que con las matemáticas es como mejor se aprende, pero cada uno lo hace de una manera.... Yo intento basarme en las matemáticas para que todos me entiendan. Son distintas formas de aprender, distintos enfoques... Y así todos lo pueden entender...

Podemos concluir, pues, que persiste la brecha entre enseñanza e investigación y que los profesores, en general, no solo no conceden importancia a la investigación para la mejora de su enseñanza sino que expresan un cierto rechazo hacia lo que califican de planteamientos "teóricos". Un rechazo que conecta, además, con concepciones espontáneas de los docentes -y, en realidad, de toda la sociedadcomo la idea de que enseñar es fácil y "basta con un buen conocimiento de la materia y algo de práctica" (Jones y Carter, 2007).

Difícilmente podrá lograrse, pues, la necesaria implicación de los profesores en la mejora de la enseñanza si estas concepciones y sus causas no son tenidas en cuenta en los procesos de reforma y en los currículos de formación del profesorado (Cronin-Jones, 1991; Russell y Martin, 2007; Vilches y Gil-Pérez, 2007). Pero para comprender dichas causas no basta con analizar las relaciones investigación/innovación atendiendo a las actitudes y comportamientos de los docentes al respecto. Es necesario también considerar la responsabilidad de los propios investigadores.

\section{¿Qué importancia conceden los investigadores a la innovación?}

Mediante un cuestionario (cuadro 2), aplicado a 29 investigadores en didáctica de las ciencias ( 16 españoles, 4 portugueses, 4 argentinos, 3 mexicanos y 2 brasileños), hemos constatado que la concepción más extendida acerca de las relaciones entre investigación e innovación es su desconexión y muy en particular (79,3\%) la que ve la innovación como aplicación de la investigación. Ello introduce otra forma de separación entre ambas, puesto que se considera que el papel de los investigadores es la construcción de conocimientos que otros (los profesores de aula) deben aplicar después.

Cuadro 2. Cuestionario para investigadores en didáctica de las ciencias

Con objeto de contribuir a comprender las interacciones entre la investigación y la innovación educativas, le agradeceríamos que exponga sus ideas al respecto:

- ¿ ¿Cómo concibe las relaciones entre investigación e innovación? 
He aquí algunos ejemplos de respuestas:

- Si los resultados de la investigación tienen un alto grado de aceptación por la comunidad científica, sería conveniente una innovación a gran escala, implicando a un gran número de profesores (...) para poner en marcha este plan de acción necesario.

- La investigación debería ser capaz de transformarse en I+D que supone una transposición didáctica sencilla, operativa, viable, adaptada a las condiciones de aula. La innovación debería vencer las "preferencias" del profesorado y tener recursos oficiales de aplicación.

- Creo que los profesores-investigadores que investigan sobre aspectos diversos de didáctica de las ciencias deberían hacer un esfuerzo para desarrollar propuestas concretas de innovación para proponer a los profesores más activos y sensibles de los centros, que por otra parte son los que también están más comprometidos en la dinámica del centro. Cuando las propuestas de innovación son concretas y funcionan, la transmisión de compañero a compañero funciona.

- Difíciles, debido a la disparidad de facto en sus intereses y finalidades. La investigación mide su calidad por parámetros diferentes a la innovación. La investigación se centra en la coherencia interna, fundamentación teórica, metodología... Mientras la innovación pretende cambiar una práctica para mejorarla.

- No siempre están relacionadas, aunque creo que sería conveniente que lo estén. Se puede innovar (bien o mal) sin hacer investigación y viceversa. La investigación permite innovar con fundamentos.

De acuerdo con estos planteamientos, algunos directores de tesis, y miembros de los tribunales que las juzgan, llegan a sostener que las investigaciones deberían limitarse a estudiar los problemas (como, por ejemplo, las preconcepciones en un determinado campo, o las diferencias entre las estrategias de resolución de problemas utilizadas por los buenos resolventes y los mediocres, etc.) sin descender a elaborar propuestas de actuación en el aula, pues ello ya no constituiría investigación, sino simple innovación.

Resulta conocida, en el mismo sentido, la política editorial de prestigiosas revistas en el campo de la educación científica, que rechazan sistemáticamente aquellos trabajos considerados de innovación, por muy fundamentados que resulten.

Todo esto recuerda una concepción muy extendida acerca de las relaciones entre ciencia y tecnología que contempla a esta última como ciencia aplicada, es decir, como mera aplicación de los conocimientos científicos (Gardner, 1994; Maiztegui et al., 2002; Ferreira-Gauchía, Vilches y Gil-Pérez, 2012). De hecho, la tecnología ha sido vista tradicionalmente como una actividad de menor estatus que la ciencia pura (Acevedo, 1996; De Vries, 1996; Cajas, 1999). Ello responde a la tradicional primacía social del trabajo intelectual frente a las actividades 
prácticas, manuales, propias de las técnicas (Medway, 1989; López Cubino, 2001). Nos tememos que tras la concepción de la innovación como aplicación de la investigación estén jugando valoraciones semejantes, que colocan la ciencia que construyen los investigadores por encima de la simple práctica docente.

Es relativamente fácil, sin embargo, cuestionar esta visión simplista de las relaciones entre ciencia y tecnología, así como entre investigación educativa y práctica docente: basta reflexionar brevemente sobre el desarrollo histórico de la ciencia y la actividad técnica (Gardner, 1994; Maiztegui et al., 2002) para comprender que esta ha precedido en milenios a la ciencia y que, por tanto, en modo alguno puede considerarse como mera aplicación de conocimientos científicos. Así mismo, los esfuerzos de innovación educativa han precedido, como sabemos bien, al desarrollo de la investigación y la han impulsado. De hecho, muchos investigadores en didáctica de las ciencias son docentes que se han acercado a la investigación tratando de buscar respuesta a los problemas surgidos en el aula, preocupados por la mejora de su enseñanza.

Nadie puede pretender hoy, por supuesto, trazar una neta separación entre ciencia y tecnología: desde la revolución industrial los tecnólogos han incorporado de forma creciente las estrategias de la investigación científica para producir y mejorar sus productos. La interdependencia de la ciencia y la tecnología ha seguido creciendo debido a su incorporación a las actividades industriales y productivas, y eso hace difícil hoy -y, al mismo tiempo, carente de interés-clasificar un trabajo como puramente científico o puramente tecnológico. Y una vez más, algo similar puede argumentarse acerca de las relaciones entre innovación e investigación educativas. Por ejemplo, ningún análisis crítico, ninguna explicación de los problemas estudiados en una investigación, pueden darse por válidos si no van acompañados de pruebas de que, al incidir sobre las supuestas causas, se producen resultados coherentes con las hipótesis manejadas. Y ello exige intervención controlada, es decir, innovación asociada a la investigación.

La brecha entre investigación e innovación puede asociarse, pues, tanto a concepciones docentes espontáneas que ignoran la complejidad del acto educativo, como a concepciones de los investigadores que desvalorizan la innovación como simple aplicación de los conocimientos construidos por ellos e ignoran el papel fundamental de las innovaciones controladas en la construcción y validación de dichos conocimientos.

\section{¿Qué se puede y conviene hacer para superar la brecha entre investigación e innovación?}

Se trata, sin duda, de una cuestión fundamental. Los investigadores y diseñadores curriculares han expresado su frustración al enfrentarse a las dificultades de mejorar la enseñanza de la ciencia a través de reformas basadas en rigurosas investigaciones. Pero esta decepción pone de manifiesto la persistencia de una visión simplista acerca de cómo los cambios en educación pueden tener lugar: la idea de que bastaría presentar las nuevas propuestas bien fundamentadas para que fueran aceptadas y aplicadas por el profesorado. Numerosas investigaciones en el campo de la formación del profesorado han cuestionado esta optimista pero, a la vez, ingenua visión (Anderson y Mitchener, 1994; Bell, 1998; Jones y Carter, 2007) y han llegado al convencimiento de que los profesores no aplicarán las recomendaciones de la investigación si no participan, como auténticos protagonistas, en el proceso de investigación/innovación (Briscoe, 1991 ; Kyle et al., 1991 ; Pekarek, Krockover y Shepardson, 
1996; Anderson y Helms, 2001 Gil-Pérez y Vilches, 2004b; Roth, 2007; Wallace y Loughran, 2012; Taylor, Taylor y Luitel, 2012).

En otras palabras, la estrategia que parece potencialmente más fructífera para favorecer las innovaciones fundamentadas consistiría en implicar al profesorado en la tarea colectiva de investigación de los problemas de enseñanza/ aprendizaje de las ciencias que les plantea su actividad docente (Vilches y Gil-Pérez, 2007). Y debemos insistir en que ello no constituye únicamente un requisito para la mejora de la enseñanza, sino también una forma de potenciar investigaciones relevantes en torno a los problemas que plantea el proceso de enseñanza/aprendizaje de las ciencias.

Esta estrategia requiere la superación de las concepciones erróneas acerca de las relaciones entre investigación e innovación educativas contempladas en general, tanto por los profesores como por los investigadores, como dos actividades muy diferentes que "no deberían confundirse". Se trata de algo esencial que deberá ser tenido en cuenta, muy particularmente, en la formación inicial del profesorado de los diferentes niveles educativos. Una formación que ha de ser planteada no solo para incorporar nuevos profesores y profesoras al sistema educativo, sino para contribuir a la necesaria renovación de la enseñanza respondiendo a los serios problemas detectados en la educación por los resultados de las evaluaciones internacionales y los informes de comisiones de expertos como, por ejemplo, el Informe Rocard (Rocard et al., 2007) o los National Science Education Standards (National Academy of Sciences, 1995), centrados en la educación científica.

Se trata, claro está, de una propuesta extremadamente exigente desde muchos puntos de vista (incluido el presupuestario), que obliga a llevar a cabo una profunda reconsideración del papel de la educación en nuestras sociedades y, muy concretamente de las condiciones de trabajo de los docentes, para hacer posible su efectiva implicación en el proceso de investigación/innovación (Gil-Pérez y Vilches, 2004b; Vilches y Gil-Pérez, 2007). Pero estamos convencidos de que es algo que se impondrá en la medida en que se comprenda que el desarrollo individual y social requiere proporcionar a todos los ciudadanos una educación de calidad a lo largo de toda su vida, incluyendo, muy en particular, la necesaria alfabetización científica para su participación en la toma de decisiones fundamentada, frente a los problemas que afectan a la humanidad (Delors, 1996; Gil-Pérez, Sifredo, Valdés y Vilches, 2005). Algo que la actual situación de emergencia planetaria convierte en una exigencia de supervivencia de la especie humana (Vilches y Gil, 2003; 2009; 2011 ). Como advierten Taylor, Taylor y Luitel (2012), "the world is wracked by crises of unparalleled proportions, forcing us to rethink the fundamentals of our lives". Necesitamos, pues, una profunda renovación de la educación científica para hacer posible la participación fundamentada de la ciudadanía en el diseño de un futuro sostenible y satisfactorio para todos. Y ello exige una más estrecha vinculación de la investigación e innovación, superando las barreras y malentendidos actuales. 


\section{Referencias bibliográficas}

Acevedo, J.A. (1996). La tecnología en las relaciones CTS. Una aproximación al tema. Enseñanza de las Ciencias 14(1), 35-44.

Anderson, C.W. (2007). Perspectives on Science Learning. En: S.K. Abell y N.G. Lederman (2007). Handbook on Research on Science Education (pp. 3-30). Nueva York: Routledge.

Anderson, R.D. y Helms, J.V. (2001). The Ideal of Standards and the reality of Schools: Needed Research. Journal of Research in Science Teaching 38(1), 3-16.

Anderson, R.D. y Mitchener, C. P. (1994). Research on science teacher education. En: D.L. Gabel (ed.). Handbook of Research on Science Teaching Education. Nueva York: Macmillan Pub. Co.

Bell, B. (1998). Teacher development in science education. En: B.J. Fraser y K.G. Tobin (eds). International Handbook of Science Education. Dordrecht: Kluber.

Briscoe, C. (1991). The dynamic interactions among beliefs, role metaphors and teaching practices. A case study of teacher change. Science Education 75(2), 185-199.

Cajas, F. (1999). Public Understanding of Science: Using technology to Enhance School Science in Everyday Life. International Journal of Science Education 21 (7), 765-773.

Cronin-Jones L.L. (1991). Science teaching beliefs and their influence on curriculum implementation: two case studies, Journal of Research in Science Teaching 38(3), 235-250.

De Vries, M. (1996). Technology Education: Beyond the 'Technology is Applied Science' Paradigm (Guest Article). Journal of Technology Education 8(1), 7-15.

Delors, J. (Coord.) (1996). La educación encierra un tesoro. Informe a la UNESCO de la Comisión Internacional sobre la educación para el siglo XXI. Madrid: Santillana. Ediciones Unesco.

Gardner, P.L. (1994). Representations of the relationship between Science and Technology in the curriculum. Studies in Science Education, 24, 1-28.

Ferreira-Gauchía, C.; Vilches, A. y Gil-Pérez, D. (2012). Concepciones docentes acerca de la naturaleza de la tecnología y de las relaciones ciencia, tecnología, sociedad y ambiente en la educación tecnológica. Enseñanza de las Ciencias 30(2), 253-272.

Gil-Pérez, D.; Carrascosa, J. y Martínez, J. (2000). Una disciplina emergente y un campo específico de investigación. En: J. Perales y P. Cañal (eds.). Didáctica de las ciencias experimentales. Alcoy: Marfil. 
Gil-Pérez, D.; Sifredo, C., Valdés, P. y Vilches, A. (2005). ¿Cuál es la importancia de la educación científica en la sociedad actual? En: D. Gil-Pérez; B. Macedo; J. Martínez Torregrosa; C. Sifredo; P. Valdés y A. Vilches (eds.). ¿Cómo promover el interés por la cultura científica? Una propuesta didáctica fundamentada para la educación científica de jóvenes de 15 a 18 años (pp. 15-28). Santiago: Orealc/Unesco.

Gil-Pérez, D. y Vilches, A. (2004a). ¿ Qué relación existe entre investigación e innovación en la educación científica? Necesidad de un serio debate y reorientación. Actas de los XXI Encuentros de Didáctica de las Ciencias Experimentales (pp. 533-539). Servicio Editorial de la Universidad del País Vasco.

Gil-Pérez, D. y Vilches, A. (2004b). La formación del profesorado de ciencias de secundaria... y de universidad. La necesaria superación de algunos mitos bloqueadores. Educación en Química 15(1), 43-58.

Jones, M.G. y Carter, G. (2007). Science Teacher Attitudes and Beliefs. En: S.K. Abell y N.G. Lederman (2007). Handbook on Research on Science Education (pp. 1067-1 104). Nueva York: Routledge.

Kyle, W.C.; Linn, M.C.; Bitner, B.L.; Mitchener, C.P. y Perry, B. (1991). The Role of Research in Science Teaching: An NSTA Theme Paper. Science Education 75(4), 413-418.

López Cubino, R. (2001). El área de tecnología en Secundaria. Madrid: Narcea.

Maiztegui, A.; Acevedo, J.A.; Caamaño, A.; Cachapuz, A.; Cañal, P.; Carvalho, A.M.P.; Dumas Carré, A.; Garritz, A.; Gil, D.; González, E.; Gras-Martí, A.; Guisasola, J.; López-Cerezo, J.A.; Macedo, B.; Martínez-Torregrosa, J.; Moreno, A.; Praia, J.; Rueda, C.; Tricárico, H.; Valdés, P. y Vilches, A. (2002). Papel de la tecnología en la educación Científica: una dimensión olvidada. Revista Iberoamericana de Educación, 28, 128-155.

Medway, P. (1989). Issues in the theory and practice of technology education. Studies in Science Education, 16, 1-24.

National Academy of Sciences (1995). National Science Education Standards. Washington, DC: National Academy Press.

Pekarek, R.; Krockover, G.H. y Shepardson, D.P. (1996). The Research-Practice Gap in Science Education. Journal of Research in Science Teaching 33(2), $111-113$.

Rocard, M.; Csermely, P.; Jorde, D.; Lenzen, D.; Walwerg-Henriksson, H. y Hemmo, V. (2007). Science Education Now: A Renewed Pedagogy for the Future of Europe. European Commission. Community Research. (De libre acceso en Internet).

Roth, K.J. (2007). Science Teachers as Researchers. En: S.K. Abell y N.G. Lederman (2007). Handbook on Research on Science Education (pp. 1203-1259). Nueva York: Routledge. 
Russell, T. y Martin, A.K. (2007). Learning to Teach Science. En: S.K. Abell y N.G. Lederman. Handbook on Research on Science Education (pp. 1151 -1178). Nueva York: Routledge.

Simon, S. y Campbell, S. (2012). Teacher Learning and Professional Development in Science Education. En: B.J. Fraser; K. Tobin y C.J. McRobbie (2012). Second International Handbook of Science Education (pp. 307-321). Dordrecht: Springer.

Taylor, P.C.; Taylor, E. y Luitel, B.C. (2012). Multi-paradigmatic Transformative Research as/for Teacher Education: An Integral Perspective. En: B.J. Fraser; K. Tobin y C.J. McRobbie (2012). Second International Handbook of Science Education (pp. 373- 387). Dordrecht: Springer.

Vilches, A. y Gil-Pérez, D. (2003). Construyamos un futuro sostenible. Diálogos de supervivencia. Madrid: Cambridge University Press.

Vilches, A. y Gil-Pérez, D. (2007). La necesaria renovación de la formación del profesorado para una educación científica de calidad. Tecné, Episteme y Didaxis: TED, 22, 67-85. (Número extraordinario dedicado a los 10 años de la Revista TED).

Vilches, A. y Gil-Pérez, D. (2009). Una situación de emergencia planetaria a la que debemos y podemos hacer frente. Revista de Educación, número extraordinario, 101-122.

Vilches, A. y Gil Pérez, D. (2011). El antropoceno como oportunidad para reorientar el comportamiento humano y construir un futuro sostenible. Revista Electrónica de Enseñanza de las Ciencias (REEC) 10(3), 394-419

Wallace, J. y Loughran, J. (2012). Science Teacher Learning. En: B.J. Fraser; K. Tobin y McRobbie C.J. (2012). Second International Handbook of Science Education (pp. 295- 306). Dordrecht: Springer. 